\title{
Effect of Proton Irradiation on the Magnetic Properties of Antiferromagnet/ferromagnet Structures
}

\author{
Dong-Jun Kim ${ }^{1}$, Jin-Seok Park², Ho Jin Ryu ${ }^{3}$, Jong-Ryul Jeong ${ }^{4}$, \\ Chang-Kyu Chung ${ }^{5}$, and Byong-Guk Park ${ }^{1 *}$ \\ ${ }^{1}$ Department of Materials Science and Engineering, KAIST, Daejeon 34141, Korea \\ ${ }^{2}$ Mechanical System Engineering Department, KEPCO E\&C, Daejeon 34057, Korea \\ ${ }^{3}$ Department of Nuclear and Quantum Engineering, KAIST, Daejeon 34141, Korea \\ ${ }^{4}$ Department of Materials Science and Engineering, Graduate School of Green Energy Technology, \\ Chungnam National University, Daejeon 34134, Korea \\ ${ }^{5}$ New Power Development Project, KEPCO E\&C, Gimcheon 39960, Korea
}

(Received 22 February 2016, Received in final form 14 April 2016, Accepted 2 May 2016)

\begin{abstract}
Antiferromagnet (AFM)/ferromagnet (FM) bilayer structures are widely used in the magnetic devices of sensor and memory applications, as AFM materials can induce unidirectional anisotropy of the FM material via exchange coupling. The strength of the exchange coupling is known to be sensitive to quality of the interface of the AFM/FM bilayers. In this study, we utilize proton irradiation to modify the interface structures and investigate its effect on the magnetic properties of AFM/FM structures, including the exchange bias and magnetic thermoelectric effect. The magnetic properties of $\mathrm{IrMn} / \mathrm{CoFeB}$ structures with various $\operatorname{IrMn}$ thicknesses are characterized after they are exposed to a proton beam of $3 \mathrm{MeV}$ and $1 \sim 5 \times 10^{14}$ ions $/ \mathrm{cm}^{2}$. We observe that the magnetic moment is gradually reduced as the amount of the dose is increased. On the other hand, the exchange bias field and thermoelectric voltage are not significantly affected by proton irradiation. This indicates that proton irradiation has more of an influence on the bulk property of the FM CoFeB layer and less of an effect on the IrMn/CoFeB interface.
\end{abstract}

Keywords : exchange bias, antiferromagnet, ferromagnet, proton irradiation

\section{Introduction}

Exchange coupling between antiferromagnet (AFM) and ferromagnet (FM) layers generates a unidirectional effective magnetic field toward the FM, known as exchange bias [1]. In spintronic devices consisting of two FM layers separated by a non-magnetic spacer, exchange bias is generally utilized to pin the magnetization direction of one FM [2]. This allows the spintronic device to serve as memory and to operate under a small magnetic field. As the exchange coupling is formed at the AFM/FM interface, control of interfacial properties such as the roughness and intermixing is essential to obtain stronger exchange bias [1]. The robustness of the exchange bias upon a postannealing step can sometimes restrain the thermal stability of spintronic devices $[3,4]$. It was recently reported that

(C)The Korean Magnetics Society. All rights reserved.

*Corresponding author: Tel: +82-42-350-3330

Fax: +82-42-350-3310, e-mail: bgpark@kaist.ac.kr
AFM in an AFM/FM bilayer structure can be used as a spin-current generator or as a spin-to-charge convertor due to its large spin-orbit coupling $[5,6]$. The former is utilized during the manipulation of FM magnetization by the in-plane current via the spin-orbit torque [7, 8], while the latter allows the AFM to be an electrode in spin thermoelectric structures [9]. Spin current transmission through the AFM/FM interface is essential in these phenomena such that the formation of fine interfaces is a prerequisite for proper performance capabilities.

Control of the AFM/FM interface after the sample deposition process has not been extensively studied thus far, except in relation to annealing at a moderate temperature [10]. The exchange bias is increased by a postannealing step, most likely due to the enhanced interfacial structures. A recent report demonstrates that proton irradiation can modify the magnetic property of FM/nonmagnetic samples of $\mathrm{Co} / \mathrm{Pd}$ multilayer structures $[11,12]$. The proton irradiation to the $\mathrm{CoO} / \mathrm{Pd}$ sample causes the reduction of the $\mathrm{CoO}$ to become a metallic $\mathrm{Co}$, thus develop- 
ing an interfacial perpendicular magnetic anisotropy of the $\mathrm{Co} / \mathrm{Pd}$ multilayers.

In this study, we utilize proton irradiation on an AFM/ FM bilayer and investigate its effect on the magnetic properties of the exchange bias and on the magneto-thermoelectric properties. We observe that saturation magnetization is gradually decreased with an increase in the proton dose; however, the exchange bias and the magneto-thermoelectric properties are not significantly affected by proton irradiation. This suggests that proton irradiation has more of an influence on the bulk FM CoFeB layer and less of an influence on the $\mathrm{IrMn} / \mathrm{CoFeB}$ interface.

\section{Experiment}

Samples of $\mathrm{Co}_{32} \mathrm{Fe}_{48} \mathrm{~B}_{20}(\mathrm{CoFeB}, 8 \mathrm{~nm}) / \mathrm{IrMn}\left(t_{\mathrm{IrMn}}\right)$ bilayer structures are fabricated by means of UHV magnetron sputtering on thermally oxidized Si substrates with a base pressure of $3 \times 10^{-8}$ Torr and a sputtering pressure of 3 mTorr. The IrMn thickness, $t_{\mathrm{IrMn}}$, is varied from 10 to $20 \mathrm{~nm}$. The samples are exposed to a proton beam of 3 $\mathrm{MeV}$ and the amount of the dose is varied from $1 \times 10^{14}$ to $5 \times 10^{14}$ ions $/ \mathrm{cm}^{2}$. The experimental location is the Korea Multi-Purpose Accelerator Complex. The effects of proton irradiation on the magnetic properties of the samples are investigated in terms of the magnetic hysteresis as measured using a vibrating sample magnetometer (VSM). The magnetic thermoelectric effect of the irradiated sample is examined using a bar-shaped sample with a width of 30 $\mu \mathrm{m}$ and a length of $1 \mathrm{~mm}$, as defined by photolithography and Ar ion milling. A vertical temperature gradient in the sample is induced by a $660-\mathrm{nm}$ continuous laser with a diameter of $5 \mu \mathrm{m}$ and power of $55 \mathrm{~mW}$. The thermoelectric voltage is measured by a nanovoltmeter as a function of the magnetic field, which is applied in a direction normal to both the temperature gradient and the voltage probe. All measurements are done at room temperature.

\section{Results and Discussion}

We initially examine the magnetic properties of the $\mathrm{CoFeB}(8 \mathrm{~nm}) / \mathrm{IrMn}\left(t_{\mathrm{IrMn}}\right)$ layers prior to the proton irradiation step, as presented in Fig. 1. Here, the value of $t_{\mathrm{IrMn}}$ is 10,15 , and $20 \mathrm{~nm}$. The magnetic hysteresis loop is centered at a zero magnetic field for the sample with an IrMn thickness of $10 \mathrm{~nm}$, while for the samples with a thicker IrMn layers, the hysteresis of the FM CoFeB layer shifts to a negative field direction, demonstrating a unidirectional exchange bias field induced by the AFM IrMn layer. IrMn thinner than $15 \mathrm{~nm}$ is not sufficient to formulate AFM order, which can instill in the FM CoFeB

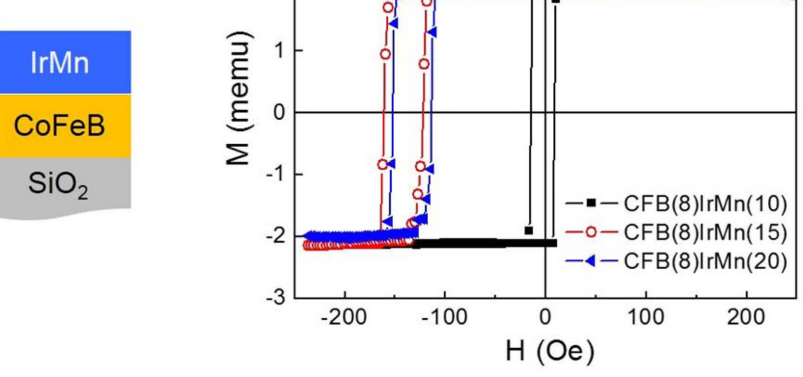

Fig. 1. (Color online) Magnetic hysteresis of the $\mathrm{CoFeB}(8$ $\mathrm{nm}) / \mathrm{IrMn}(10,15,20 \mathrm{~nm})$ samples which are measured using vibrating sample magnetometer (VSM). The shift of the hysteresis indicates the exchange bias coupling between FM $\mathrm{CoFeB}$ and AFM IrMn.

an exchange bias field [1]. Moreover, the exchange bias becomes saturated at an IrMn thickness of $15 \mathrm{~nm}$, which indicates that the exchange bias effect is dominated by the $\mathrm{AFM} / \mathrm{FM}$ interface rather than by the bulk AFM. Before we discuss the variation of the magnetic properties with the proton irradiation, we show the simulation results of a radiation damage in dpa (displacement per atom) and a penetration depth of the proton ions in a $\mathrm{Co}_{40} \mathrm{Fe}_{60}$ materials using SRIM-2008 code [13], estimating the effect of the

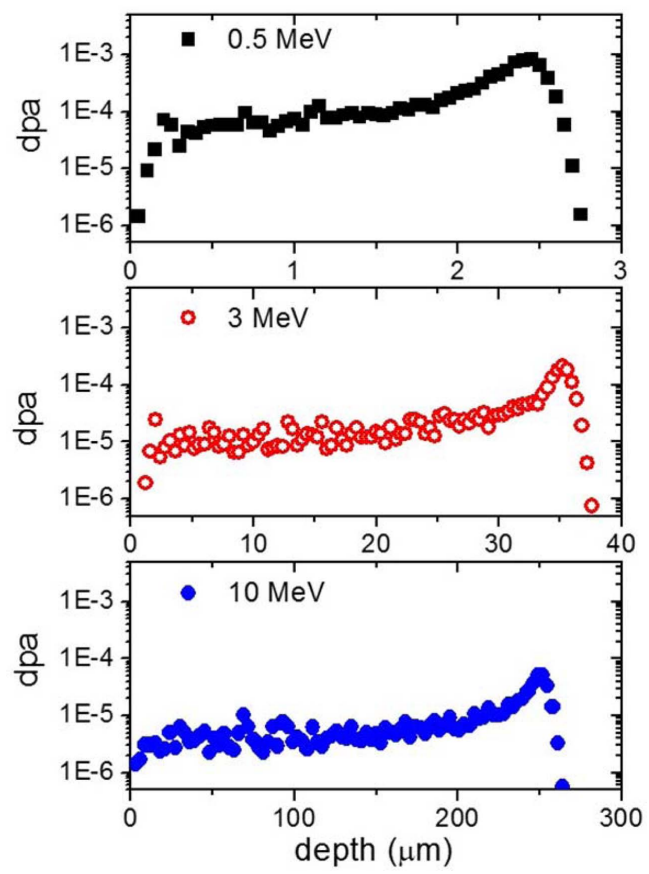

Fig. 2. (Color online) Radiation damage (dpa) and penetration depth of $\mathrm{Co}_{40} \mathrm{Fe}_{60}$ targets under $0.5 \mathrm{MeV}, 3 \mathrm{MeV}$, and $10 \mathrm{MeV}$ for proton fluence of $5 \times 10^{14}$ ions $/ \mathrm{cm}^{2}$, calculated by SRIM code. 
Table 1. Summary of damage and penetration depth depending on proton energy.

\begin{tabular}{cccc}
\hline \hline $\begin{array}{c}\text { Ion } \\
\text { Energy }\end{array}$ & $\begin{array}{c}\text { Max. } \\
\text { Damage } \\
(\mathrm{dpa})\end{array}$ & $\begin{array}{c}\text { Avg. } \\
\text { Damage } \\
(\mathrm{dpa})\end{array}$ & $\begin{array}{c}\text { Penetration } \\
\text { Depth } \\
(\mu \mathrm{m})\end{array}$ \\
\hline $500 \mathrm{keV}$ & $8.1 \times 10^{-4}$ & $1.7 \times 10^{-4}$ & 2.7 \\
$3 \mathrm{MeV}$ & $2.1 \times 10^{-4}$ & $2.7 \times 10^{-5}$ & 37 \\
$10 \mathrm{MeV}$ & $5.2 \times 10^{-5}$ & $7.6 \times 10^{-6}$ & 267 \\
\hline
\end{tabular}

proton irradiation on the materials. Figure 2 shows the dependence of the radiation damage and penetration depth on proton energy. As the acceleration energy is increased from 0.5 to $10 \mathrm{MeV}$, the penetration depth is changed from 2.7 to $267 \mu \mathrm{m}$ when the fluence was $5 \times 10^{14}$ ions/ $\mathrm{cm}^{2}$. In contrast, proton radiation damage to the materials is reduced from $1.7 \times 10^{-4}$ to $7.6 \times 10^{-6}$ for the change of proton energy from 0.5 to $10 \mathrm{MeV}$. Table 1 summarizes the variation of the penetration depth and radiation damage in a $\mathrm{Co}_{40} \mathrm{Fe}_{60}$ layer for different proton energy of $0.5,3$, $10 \mathrm{MeV}$. This demonstrates that the higher energy proton causes less radiation damage in the materials.

Next, we examine the effect of proton irradiation on the magnetic properties of the samples. The samples are exposed to a $3 \mathrm{MeV}$ proton beam at various doses, i.e., $1 \times$ $10^{14}, 3 \times 10^{14}$, and $5 \times 10^{14} \mathrm{ions} / \mathrm{cm}^{2}$. The proton irradiation results are shown in Fig. 3. For the sample with the $10 \mathrm{~nm}$ layer of IrMn, where no exchange is observed, the saturation magnetization gradually decreases as the dose amount is increased (Fig. 3a). Upon exposure to a proton beam of $5 \times 10^{14}$ ions $/ \mathrm{cm}^{2}$, the magnetization is reduced to $\sim 70 \%$ of its original value. The reduction of saturation magnetization was also observed in $\mathrm{Co} / \mathrm{Pd}$ multilayer [12], when it was exposed to a proton beam of a few hundred $\mathrm{eV}$. It was explained that the reduction of magnetization is due to the incorporation of the hydrogen in the magnetic layer [14], where the hydrogenation of the transition metals of $\mathrm{Fe}, \mathrm{Co}$, Ni causes the reduction of saturation magnetization by modification of the electronic structure. The reduction of saturation magnetization can also be due to the structural variation of the $\mathrm{IrMn} / \mathrm{CoFeB}$ sample induced by the proton irradiation. To verify this, we have measured X-ray diffraction pattern for the $\mathrm{IrMn} / \mathrm{CoFeB}$ samples exposed to proton beam with a various energy of $0,0.3$, and $5 \mathrm{MeV}$. Figure 4 shows the results, demonstrating that there is no significant different in X-ray patterns among the samples. Therefore, structural variation by the proton irradiation is less likely to be an origin of the reduction of magnetization.

Figure $3(\mathrm{~b})$ shows the proton irradiation results from the sample with the $15 \mathrm{~nm}$ IrMn layer, in which two noticeable features are shown. The first is the reduction of the saturation magnetization, as observed in the sample with the $10 \mathrm{~nm}$ IrMn layer. The second is the similar exchange bias field irrespective of the dose amount up to $5 \times 10^{14}$ ions $/ \mathrm{cm}^{2}$. These two characteristics appear to contradict each other, as one property is degraded while the other is unaffected. The results from the sample with a

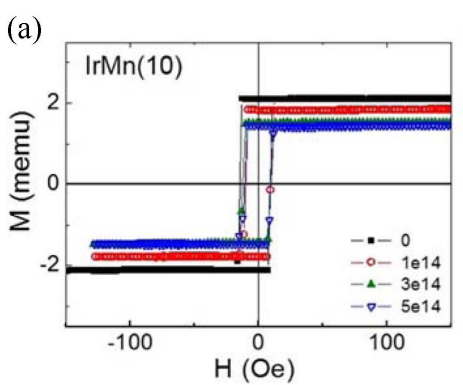

(d)

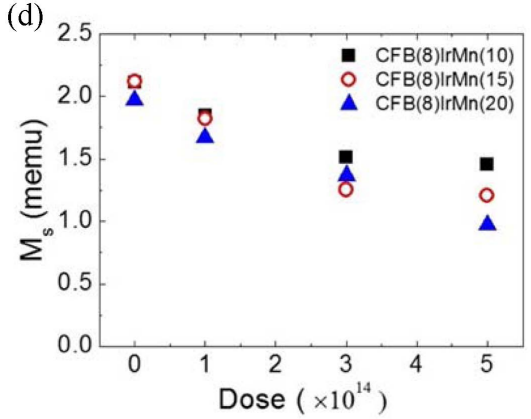

(b)

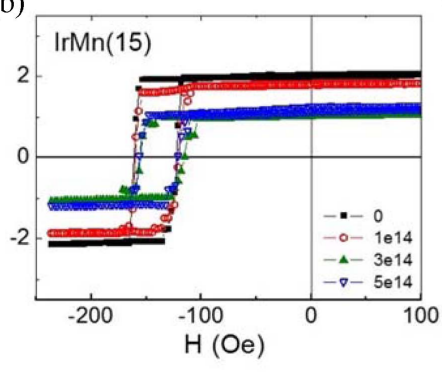

(e)

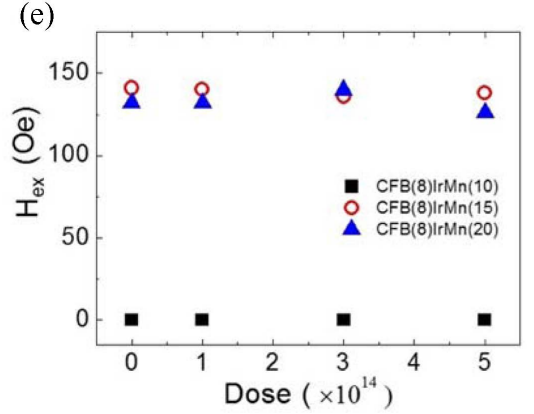

Fig. 3. (Color online) Magnetic hysteresis of the $\mathrm{CoFeB}(8 \mathrm{~nm}) / \operatorname{IrMn}\left(t_{\mathrm{IrMn}}\right)$ samples after proton irradiation of $3 \mathrm{MeV}$ and $1 \sim 5 \times 10^{14}$ ions $/ \mathrm{cm}^{2}$. (a) $t_{\mathrm{IrMn}}=10 \mathrm{~nm}$, (b) $t_{\mathrm{IrMn}}=15 \mathrm{~nm}$, (c) $t_{\mathrm{IrMn}}=20 \mathrm{~nm}$. (d, e) Summary of the dependence of the proton irradiation on the saturation magnetization, $M_{\mathrm{s}}(\mathrm{d})$ and exchange bias field, $H_{\mathrm{ex}}(\mathrm{e})$.
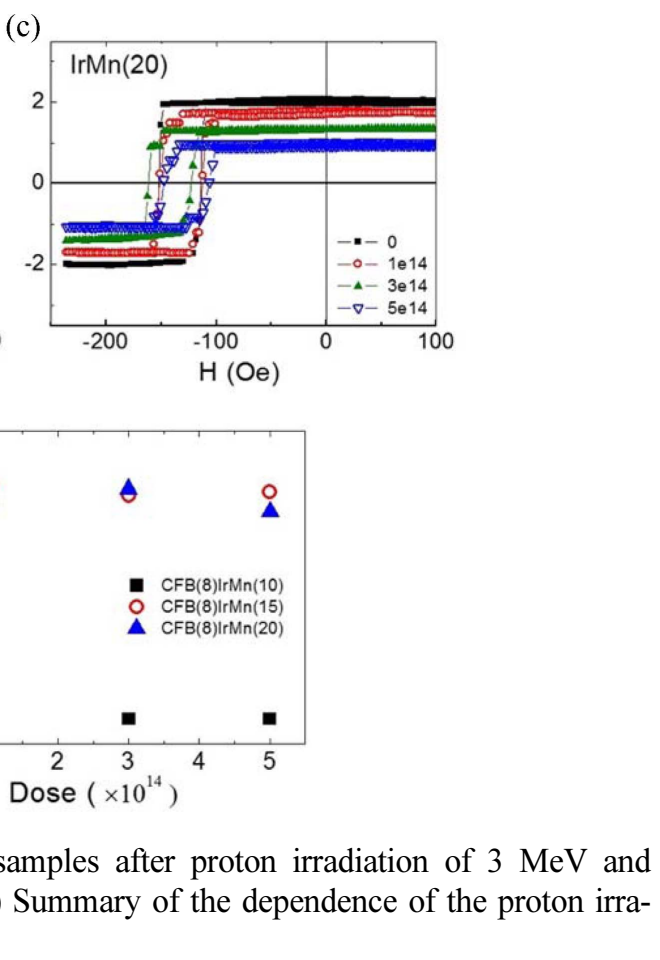


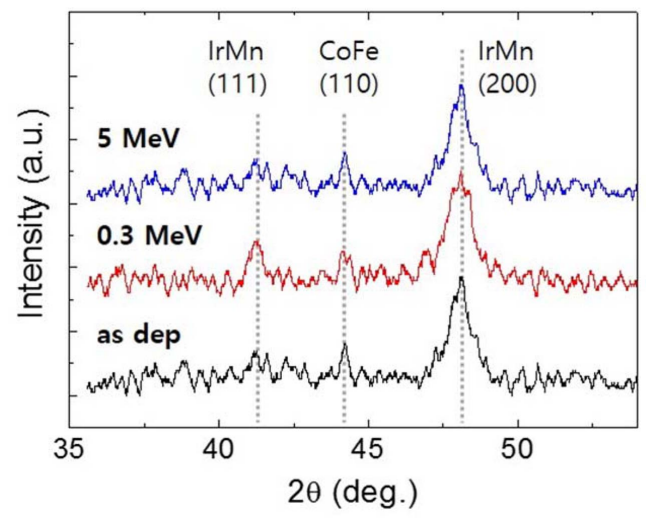

Fig. 4. (Color online) X-ray diffraction patterns of the $\mathrm{CoFeB}$ $(8 \mathrm{~nm}) / \mathrm{IrMn}(20 \mathrm{~nm})$ samples exposed to proton beam with a various energy of $0,0.3$, and $5 \mathrm{MeV}$ for proton fluence of $5 \times 10^{14}$ ions $/ \mathrm{cm}^{2}$.

$20 \mathrm{~nm}$ layer confirm the observation of reduced magnetization and similar levels of exchange bias after the proton irradiation step (Fig. 3c). Because the measured magnetization solely originates from the $\mathrm{FM} \mathrm{CoFeB}$, reducing it reveals that proton irradiation affects the internal structure of $\mathrm{CoFeB}$ and the resultant magnetic properties. On the other hand, the exchange bias relies on an alternating magnetic order in the AFM and AFM/FM interface; therefore, the unaffected exchange bias demonstrates that the IrMn and $\mathrm{IrMn} / \mathrm{CoFeB}$ interfaces are less sensitive to proton irradiation. Figs. 3(d) and 3(e) summarize the dependence of the saturation magnetization and the exchange bias field on the degree of proton exposure.

In order to confirm the effect of the proton irradiation step on the $\mathrm{IrMn} / \mathrm{CoFeB}$ bilayer, we perform an independent test via magneto-thermoelectric measurements [9, 15]. As shown in the schematic of the measurement in Fig. 5(a), when a temperature gradient is applied in the AFM/FM bilayer, transverse voltage is generated by the inverse spin Hall effect of the thermally injected spin current and by the anomalous Nernst effect [15]. The inverse spin Hall effect is determined by the spin Hall angle of the AFM IrMn and the injected spin current from the $\mathrm{IrMn} / \mathrm{CoFeB}$ interfaces, while the anomalous Nernst effect alone dominates in the FM CoFeB case. Therefore, the latter is expected to be weakened by proton irradiation as the saturation magnetization is reduced.

Figs. 5(b) (d) illustrate the dependence of the magnetothermoelectric effect on the proton irradiation for samples with different IrMn thicknesses. Note that a thermal gradient is induced by laser illumination in our experiment. The thermoelectric voltage is measured as a function of the inplane magnetic field applied in the transverse direction of the voltage contact. The thermoelectric voltage of the IrMn/CoFeB samples changes its sign upon a reversal of the magnetic field (or magnetization), which demonstrates the magnetic origin of the observed thermoelectric signal, in this case the inverse spin Hall effect and the anomalous Nernst effect. It is important to note that the shifts shown

(a)

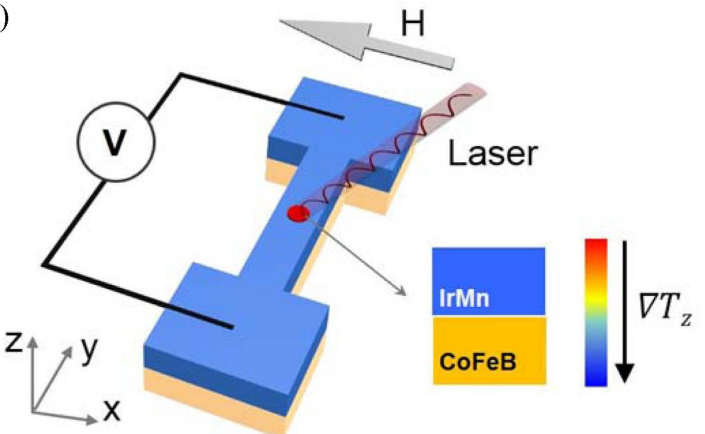

(b)

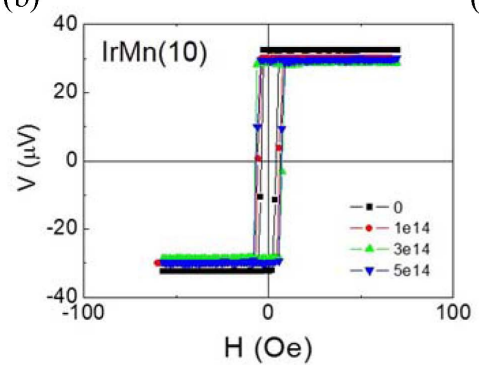

(c)

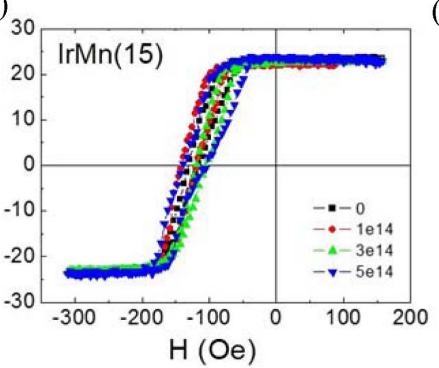

(d)

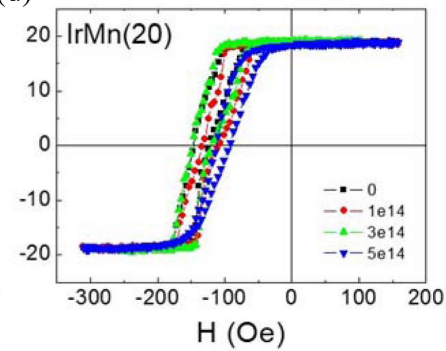

Fig. 5. (Color online) (a) Schematic of spin thermoelectric measurement. Magnetic field dependence of the spin thermoelectric voltage for the CoFeB $(8 \mathrm{~nm}) / \operatorname{IrMn}\left(t_{\mathrm{IrMn}}\right)$ samples (a) $t_{\mathrm{IrMn}}=10 \mathrm{~nm}$, (b) $t_{\mathrm{IrMn}}=15 \mathrm{~nm}$, (c) $t_{\mathrm{IrMn}}=20 \mathrm{~nm}$ after proton irradiation. 
in the graphs in Figs. 5(c) and 5(d) can be attributed to exchange bias, as discussed in Fig. 5. Interestingly, the thermoelectric voltage is not affected by proton irradiation. Because the anomalous Nernst effect depends on the magnetization of the FM CoFeB, the total thermoelectric voltage is expected to be decreased. On the other hand, the inverse spin Hall effect is determined by spin Hall angle of the IrMn layer and thermally-induced spin current, which depend on the properties of IrMn and AFM/FM interface, which are not necessarily degraded with saturation magnetization. The unaffected exchange bias (Figs. 3 and 5) shows the robustness of the IrMn layer against the proton irradiation, at least within our experimental conditions. Therefore, the insensitive magneto-thermoelectric voltage implies that the proton irradiation may modify the properties of the AFM IrMn or AFM/FM interface to enhance the inverse spin Hall effect via the modulation spin Hall angle of AFM IrMn or AFM/FM interface properties. However, in order to confirm this scenario, further studies which include the determination of spin Hall angle of IrMn and spin mixing conductance of $\mathrm{IrMn} / \mathrm{CoFeB}$ structures are required. Nevertheless, the results of this study of thermoelectric effects suggest that the effects of proton irradiation on the $\mathrm{IrMn} / \mathrm{CoFeB}$ interface and on the properties of the IrMn layer are negligible.

\section{Conclusion}

We investigate the effects of proton irradiation on the magnetic properties of an $\mathrm{IrMn} / \mathrm{CoFeB}$ bilayer by measuring the exchange bias field and the magneto-thermoelectric voltage. Samples of the $\mathrm{IrMn} / \mathrm{CoFeB}$ structures with various IrMn thicknesses are exposed to a proton beam of 3 $\mathrm{MeV}$ and $1 \sim 5 \times 10^{14}$ ions $/ \mathrm{cm}^{2}$. The saturation magnetization is gradually decreased with an increase in the proton dose, while the exchange bias and magneto-thermoelectric effect are not greatly affected. These findings suggest that proton irradiation has more of an influence on the bulk FM $\mathrm{CoFeB}$ and less of an effect on the $\mathrm{IrMn} / \mathrm{CoFeB}$ interface.

\section{Acknowledgements}

This work was supported by the Nuclear Power Core Technology Development Program of the Korea Institute of Energy Technology Evaluation and Planning (KETEP), granted financial resource from the Ministry of Trade, Industry \& Energy, Republic of Korea (No. 20131510101680). This work was also supported by National Research Foundation of Korea (NRF-2014R1A2A1A11051344). We acknowledge a support of Korea Multi-purpose Accelerator Complex for proton irradiation.

\section{References}

[1] J. Nogués and I. K. Schuller, J. Magn. Magn. Mater. 192, 203 (1999).

[2] B. Dieny, J. Magn. Magn. Mater. 136, 335 (1994).

[3] J. H. Lee, H. D. Jeong, H. Kyung, C. S. Yoon, C. K. Kim, B. G. Park, and T. D. Lee, J. Appl. Phys. 91, 217 (2002).

[4] J. H. Lee, S. J. Kim, C. S. Yoon, C. K. Kim, B. G. Park, and T. D. Lee, J. Appl. Phys. 92, 6241 (2002).

[5] W. Zhang, M. B. Jungfleisch, W. Jiang, J. E. Pearson, A. Hoffmann, F. Freimuth, and Y. Mokrousov, Phys. Rev. Lett. 113, 196602 (2014).

[6] J. B. S. Mendes, R. O. Cunha, O. Alves Santos, P. R. T. Ribeiro, F. L. A. Machado, R. L. Rodríguez-Suárez, A. Azevedo, and S. M. Rezende, Phys. Rev. B 89, 140406(R) (2014).

[7] I. M. Miron, K. Garello, G. Gaudin, P.-J. Zermatten, M. V. Costache, S. Auffret, S. Bandiera, B. Rodmacq, A. Schuhl, and P. Gambardella, Nature 476, 189 (2011).

[8] Luqiao Liu, Chi-Feng Pai, Y. Li, H. W. Tseng, D. C. Ralph, and R. A. Buhrman, Science 336, 555 (2012).

[9] K. Uchida, S. Takahashi, K. Harii, J. Ieda, W. Koshibae, K. Ando, S. Maekawa, and E. Saitoh, Nature 455, 778 (2008).

[10] H. Xi, B. Bian, Z. Zhuang, D. E. Laughlin, and R. M. White, IEEE Trans on Magnetics 30, 2644 (2000).

[11] S. Kim, S. Lee, J. Ko, J. Son, M. Kim, S. Kang, and J. Hong, Nature Nanotech. 7, 567 (2012).

[12] S. Kim, S. Lee, and J. Hong, ACS Nano 84698 (2014).

[13] J. F. Ziegler, M. D. Ziegler, and J. P. Biersack, Nuclear Instruments and Methods in Physics Research Section B: Beam Interactions with Materials and Atoms 268, 1818 (2010).

[14] N. Ishimatsu, T. Shichijo, Y. Matsushima, H. Maruyama, Y. Matsuura, T. Tsumuraya, T. Shishidou, T. Oguchi, N. Kawamura, M. Mizumaki, T. Matsuoka, and K. Takemura, Phys. Rev. B 86, 104430 (2012).

[15] K.-D. Lee, D.-J. Kim, H. Y. Lee, S.-H. Kim, J.-H. Lee, K.-M. Lee, J.-R. Jeong, K.-S. Lee, H.-S. Song, J.-W. Sohn, S.-C. Shin, and B.-G. Park, Scientific Reports 5, 10249 (2015). 